\title{
Construction of the Consistent Estimate of the Spectral Density of a Discrete-Time Homogeneous Stable Random Fields
}

\author{
Nikolay Demesh and Sergey Chekhmenok \\ Belarus State University, Minsk
}

\begin{abstract}
In this paper, we construct an estimate of the spectral density of a discrete-time homogeneous symmetric $\alpha$-stable random fields using $2 \pi$ periodic spectral windows, and prove its weak consistency.
\end{abstract}

Keywords: Homogeneous Stable Fields, Spectral Density Estimate.

\section{Introduction}

Recently the usage of methods of spectral analysis of stochastic processes and random fields in many areas of scientific research has been considerably extended. A particular attention has been focused on methods of spectral analysis of discrete-time stationary processes and homogeneous fields. There are many monographes and papers which are devoted to the research in this area: Brillinger (1975); Leonenko and Ivanov (1989); Skorokhod (1989); Demesh and Chekhmenok (2004b). The most represented topic in the literature is the spectral analysis of processes and fields with finite second-order moments. Problems dealing with the research of random processes and fields which do not have second-order moments have become of particular importance. Especially it concerns $\alpha$ stable random processes and fields, $0<\alpha<2$, which do not have second-order moments and only Gaussian processes and fields $(\alpha=2)$ have finite variance among them. As it follows from Zolotarev (1986), in case where $\alpha<2$ there are finite moments only of order $p, 0<p<\alpha$. Therefore traditional methods cannot be applied for solving practical problems and the development of a special theory is required.

\section{Assumptions and Notation}

Let us give our basic notations to be used in the article. Denote by $N=\{1,2, \ldots\}$ the set of natural numbers, $Z=\{0, \pm 1, \pm 2, \ldots\}$ the set of integer numbers, $\Pi^{n}=[-\pi, \pi]^{n}$, $P^{n}=\left[-\tau_{1}, \tau_{1}\right] \times\left[-\tau_{2}, \tau_{2}\right] \times \ldots \times\left[-\tau_{n}, \tau_{n}\right]$ an integer lattice of $n$-dimensional parallelepiped where $\tau_{j} \in N, j=\overline{1, n} . T=\left(T_{1}, \ldots, T_{n}\right)$ the $n$-dimensional vector having $\left.T_{j}=2 \tau_{j}+1, j=\overline{1, n} ; N_{T}=T_{1} \times \ldots \times T_{n} ;<a, b\right\rangle$ the inner product of the vectors $a=\left(a_{1}, \ldots, a_{n}\right)$ and $b=\left(b_{1}, \ldots, b_{n}\right)$. The notation $T \longrightarrow \infty$ means that $T_{j} \longrightarrow \infty$, $j=\overline{1, n}$, we write $\|a\|=(\langle a, a\rangle)^{\frac{1}{2}}$; the notation $x_{T} \cong y_{T}$ means that $x_{T}-y_{T} \longrightarrow 0$ as $T \longrightarrow \infty$; the notation $\lambda^{(1)} \neq \lambda^{(2)}(\bmod 2 \pi)$ where $\lambda^{(1)}=\left(\lambda_{1}^{(1)}, \lambda_{2}^{(1)}, \ldots, \lambda_{n}^{(1)}\right)$, $\lambda^{(2)}=\left(\lambda_{1}^{(2)}, \lambda_{2}^{(2)}, \ldots, \lambda_{n}^{(2)}\right) \in \Pi^{n}$ means that $\lambda_{j}^{(1)} \neq \lambda_{j}^{(2)}(\bmod 2 \pi), j=\overline{1, n}$; the notation $T^{(1)} \geq T^{(2)}$ where $T^{(1)}=\left(T_{1}^{(1)}, T_{2}^{(1)}, \ldots, T_{n}^{(1)}\right), T^{(2)}=\left(T_{1}^{(2)}, T_{2}^{(2)}, \ldots, T_{n}^{(2)}\right)$ means that $T_{j}^{(1)} \geq T_{j}^{(2)}, j=\overline{1, n}$, the notation $T^{(\max )}=\max \left\{T^{(1)}, T^{(2)}\right\}$ where $T^{(1)}=$ 
$\left(T_{1}^{(1)}, T_{2}^{(1)}, \ldots, T_{n}^{(1)}\right), T^{(2)}=\left(T_{1}^{(2)}, T_{2}^{(2)}, \ldots, T_{n}^{(2)}\right)$ means that $T_{j}^{(\max )}=\max \left\{T_{j}^{(1)}, T_{j}^{(2)}\right\}$, $j=\overline{1, n}$.

Denote by $D(p)=\int_{-\infty}^{\infty}|u|^{-1-p}(1-\cos (u)) d u$, $F(p, \alpha)=\int_{-\infty}^{\infty}|u|^{-1-p}\left(1-e^{-|u|^{\alpha}}\right) d u, c_{\alpha}=\frac{1}{\alpha \pi} \int_{0}^{\pi}|\cos (u)|^{\alpha} d u$, and

$$
k(p, \alpha)=\frac{D(p)}{F(p, \alpha)\left(c_{\alpha}\right)^{\frac{p}{\alpha}}} .
$$

Let $h_{T}(t), t=\left(\frac{t_{1}}{\tau_{1}}, \frac{t_{2}}{\tau_{2}}, \ldots, \frac{t_{n}}{\tau_{n}}\right)$, be an $n$-dimensional data window,

$$
\begin{aligned}
H^{(T)}(\lambda) & =\sum_{t \in P^{n}} h_{T}(t) \exp (-i<t, \lambda>), \\
A_{T} & =\left[\int_{\Pi^{n}}\left|H^{(T)}(\lambda)\right|^{\alpha} d \lambda\right]^{-\frac{1}{\alpha}} .
\end{aligned}
$$

Let us consider numerical sequences $M_{T_{j}}, M_{T_{j}} \in N$, and $L_{T_{j}}, L_{T_{j}} \in N, j=\overline{1, n}$, where $M_{T_{j}} \longrightarrow \infty$ as $T_{j} \rightarrow \infty$ but $\frac{M_{T_{j}}}{T_{j}} \longrightarrow 0$ as $T_{j} \rightarrow \infty, j=\overline{1, n} ; L_{T_{j}} \longrightarrow \infty$, $\frac{M_{T_{j}}}{L_{T_{j}}} \longrightarrow 0, \frac{L_{T_{j}}}{T_{j}} \longrightarrow 0$ as $T_{j} \longrightarrow \infty$ for all $j=\overline{1, n}$. Denote by

$$
\begin{gathered}
M_{T}=\prod_{j=1}^{n} M_{T_{j}}, \\
L_{T}=\prod_{j=1}^{n} L_{T_{j}} .
\end{gathered}
$$

Let $w_{T}(l)=w\left(\frac{l_{1}}{M_{T_{1}}}, \frac{l_{2}}{M_{T_{2}}}, \ldots, \frac{l_{n}}{M_{T_{n}}}\right)$ be a $n$-dimensional correlation window, $l=$ $\left(l_{1}, l_{2}, \ldots, l_{n}\right)$, where $w(x), x \in R^{n}$, satisfies the following conditions:

$$
\begin{gathered}
\sup _{x \in R^{n}} w(x)=w(0)=1, \\
0 \leq w(x) \leq 1, x \in R^{n}, \\
\int_{R^{n}} w^{2}(x) d x<\infty,
\end{gathered}
$$

and $W_{T}(\nu), \nu \in \Pi^{n}$, is a nonnegative spectral data window of the form

$$
W_{T}(\nu)=\frac{1}{(2 \pi)^{n}} \sum_{l_{1}=-M_{T_{1}}}^{M_{T_{1}}} \sum_{l_{2}=-M_{T_{2}}}^{M_{T_{2}}} \ldots \sum_{l_{n}=-M_{T_{n}}}^{M_{T_{n}}} w_{T}(l) \exp (-i<\nu, l>), \nu \in \Pi^{n} .
$$

Examples of spectral data windows can be found in Brillinger (1975); Trush (1999). 


\subsection{Main Results}

The definition of a symmetric $\alpha$-stable random field is due to Nolan (1988). Let $0<$ $\alpha \leq 2$ and $B \subset Z^{n}$. A complex-valued random field $X(t), t \in B$, is called $\alpha$-stable if for any $m \geq 1, a_{j} \in C, t^{(j)} \in B, j=\overline{1, m}$, every linear combination $\sum_{j=1}^{m} a_{j} X\left(t^{(j)}\right)$ is a symmetric $\alpha$-stable random variable. Each $\alpha$-stable random field $X(t), t \in B$, has a representation as a stochastic integral: there exists a measurable space $(\Omega, F, P)$ and a collection $\{f(t, \cdot): t \in B\} \subset L^{\alpha}(\Omega, F, P)$ such that $X(t)=\int f(t, u) \zeta(d u)$. Then $\zeta$ is the $\alpha$-stable random measure generated by $P$. A method for constructing $\zeta$ is described in Hardin (1982).

Consider a class of symmetric $\alpha$-stable fields which are called harmonizable random fields. Let $X(t), t \in Z^{n}$, be a homogeneous symmetric $\alpha$-stable $(0<\alpha<2)$ discretetime random field having the spectral representation of the form

$$
X(t)=\int_{\Pi^{n}} \exp (i<t, \lambda>) d \xi(\lambda)
$$

where $t=\left(t_{1}, t_{2}, \ldots, t_{n}\right), \lambda=\left(\lambda_{1}, \lambda_{2}, \ldots, \lambda_{n}\right)$, and where $\xi(\lambda)$ is $\alpha$-stable random field with independent increments such that $\left[E|d \xi(\lambda)|^{p}\right]^{\frac{\alpha}{p}}=C(p, \alpha) \varphi(\lambda) d \lambda$ for $p \in(0, \alpha)$. The constant $C(p, \alpha)$ depends on $p$ and $\alpha$ only, the function $\varphi(\lambda), \lambda \in R^{n}$, is a nonnegative even $2 \pi$-periodic function in each argument on $R^{n}$. This function is called spectral density of $X(t), t \in Z^{n}$. If $\alpha=2$, the field $X(t), t \in Z^{n}$, is Gaussian, the function $\varphi(\lambda)$, $\lambda \in \Pi^{n}$, is the "usual" spectral density and a standard spectral analysis is then applied. If $0<\alpha<2$, the function $\varphi(\lambda), \lambda \in \Pi^{n}$, does not represent spectral density in the usual sense. However, it was shown in Nolan (1988) that for the linear prediction and filtering the role of this function is quite similar to that played by the spectral density function in the second-order fields.

Let

$$
\left\{X\left(t_{1}, t_{2}, \ldots, t_{n}\right), t=\left(t_{1}, t_{2}, \ldots, t_{n}\right) \in P^{n}\right\}
$$

be $N_{T}$ observations of the field $X(t), t \in Z^{n}$, on $P^{n}$.

Definition 1. The statistic

$$
d_{T}(\lambda)=A_{T} \sum_{t \in P^{n}} h_{T}(t) X(t) \cos (<t, \lambda>), \lambda \in \Pi^{n},
$$

is called the finite modified Fourier transform of observations (8) of the field $X(t), t \in Z^{n}$, where $h_{T}(t), t \in Z^{n}$, is an $n$-dimensional data window and $A_{T}$ is given by (3).

Def in ition 2. The statistic

$$
I_{T}(\lambda)=k(p, \alpha)\left|d_{T}(\lambda)\right|^{p}, \lambda \in \Pi^{n},
$$

is called the extended periodogram of observations (8) of the field $X(t), t \in Z^{n}$ where $d_{T}(\lambda), \lambda \in \Pi^{n}$, is given by (9) and $k(p, \alpha)$ is defined by $(1)$.

As an estimate of the function $[\varphi(\lambda)]^{\frac{p}{\alpha}}, \lambda \in \Pi^{n}$, we consider a smoothed periodogram of the form

$$
\widehat{f}_{T}(\lambda)=\int_{\Pi^{n}} W_{T}(\nu) I_{T}(\lambda+\nu) d \nu, \lambda \in \Pi^{n},
$$


where $\nu=\left(\nu_{1}, \nu_{2}, \ldots, \nu_{n}\right), d \nu=d \nu_{1} d \nu_{2} \ldots d \nu_{n}$, and where $I_{T}(\lambda), \lambda \in \Pi^{n}$, is given by (10) and the spectral window $W_{T}(\nu), \nu \in \Pi^{n}$, is defined by (7). Let us consider the statistic

$$
\widehat{\varphi}_{T}(\lambda)=\left[\widehat{f}_{T}(\lambda)\right]^{\frac{\alpha}{p}}, \lambda \in \Pi^{n},
$$

as an estimate of the spectral density $\varphi(\lambda), \lambda \in \Pi^{n}$.

Defin ition 3. A set of even $2 \pi$-periodic in each argument functins $G_{T}(\lambda), \lambda \in$ $\Pi^{n}$, is called a kernel on $\Pi^{n}$ if it satisfies the following conditions:

$$
\begin{gathered}
\int_{\Pi^{n}} G_{T}(\lambda) d \lambda=1 \text { for any } T=\left(T_{1}, T_{2}, \ldots, T_{n}\right), T_{j}=1,2, \ldots j=\overline{1, n}, \\
\qquad \int_{\Pi^{n} \backslash\{\|\lambda\| \leq \delta\}} G_{T}(\lambda) d \lambda \longrightarrow 0 \text { for any fixed } \delta \in(0, \pi) \text { as } T \rightarrow \infty .
\end{gathered}
$$

Theor e m. Assume that the spectral density $\varphi(\lambda), \lambda \in \Pi^{n}$, of the field $X(t), t \in$ $Z^{n}$, is positive and continuous on $\Pi^{n}$. Let $W_{T}(\nu), \nu \in \Pi^{n}$, and $\left|H_{T}(\lambda)\right|^{\alpha}=$ $=\left|A_{T} H^{(T)}(\lambda)\right|^{\alpha}, \lambda \in \Pi^{n}$, be a kernel on $\Pi^{n}$ where $A_{T}$ and $H^{(T)}(\lambda)$ are defined by (3) and (2), respectively. Assume also that

$$
\int_{\Pi^{n}}\left|H_{T}\left(\lambda^{(1)}-\mu\right) H_{T}\left(\lambda^{(2)}-\mu\right)\right|^{\frac{\alpha}{2}} d \mu \underset{T \rightarrow \infty}{\longrightarrow} 0
$$

for all $\lambda^{(1)}, \lambda^{(2)} \in \Pi^{n}, \lambda^{(1)} \neq \lambda^{(2)}(\bmod 2 \pi)$. Then

$$
\widehat{\varphi}_{T}\left(\lambda^{(0)}\right) \underset{T \rightarrow \infty}{\stackrel{P}{\longrightarrow}} \varphi\left(\lambda^{(0)}\right)
$$

for all $\lambda^{(0)} \in \Pi^{n}$, where $\widehat{\varphi}_{T}(\lambda), \lambda \in \Pi^{n}$, is given by (12).

\section{Appendix}

Proof of Theorem.

Let us consider the mathematical expectation of the estimation (11) and show that $E \widehat{f}_{T}\left(\lambda^{(0)}\right) \longrightarrow\left[\varphi\left(\lambda^{(0)}\right)\right]^{\frac{p}{\alpha}}$ as $T \longrightarrow \infty$, That is for any $\epsilon>0$ there exists $T(\epsilon)$, that $\left|E \widehat{f}_{T}\left(\lambda^{(0)}\right)-\left[\varphi\left(\lambda^{(0)}\right)\right]^{\frac{p}{\alpha}}\right| \leq \epsilon$ for all $T \geq T(\epsilon)$. As it follows from Trush and Orlova (1994), we have

$$
E I_{T}(\lambda)=\left[\psi_{T}(\lambda)\right]^{\frac{p}{\alpha}}, \lambda \in \Pi^{n}
$$

where

$$
\psi_{T}(\lambda)=\int_{\Pi^{n}}\left|H_{T}(\nu)\right|^{\alpha} \varphi(\lambda+\nu) d \nu, \lambda \in \Pi^{n} .
$$

As far as the function $W_{T}(\nu), \nu \in \Pi^{n}$, is a kernel on $\Pi^{n}$, then by (15) and (16), we obtain

$$
\left|E \widehat{f}_{T}\left(\lambda^{(0)}\right)-\left[\varphi\left(\lambda^{(0)}\right)\right]^{\frac{p}{\alpha}}\right|=\left|E \int_{\Pi^{n}} W_{T}(\nu) I_{T}\left(\lambda^{(0)}+\nu\right) d \nu-\left[\varphi\left(\lambda^{(0)}\right)\right]^{\frac{p}{\alpha}}\right|=
$$




$$
\begin{aligned}
& =\left|\int_{\Pi^{n}} W_{T}(\nu)\left[\psi_{T}\left(\lambda^{(0)}+\nu\right)\right]^{\frac{p}{\alpha}} d \nu-\left[\varphi\left(\lambda^{(0)}\right)\right]^{\frac{p}{\alpha}}\right|= \\
& =\left|\int_{\Pi^{n}} W_{T}(\nu)\left[\left[\psi_{T}\left(\lambda^{(0)}+\nu\right)\right]^{\frac{p}{\alpha}}-\left[\varphi\left(\lambda^{(0)}\right)\right]^{\frac{p}{\alpha}}\right] d \nu\right| \leq \\
& \leq \int_{\Pi^{n}} W_{T}(\nu)\left|\left[\psi_{T}\left(\lambda^{(0)}+\nu\right)\right]^{\frac{p}{\alpha}}-\left[\varphi\left(\lambda^{(0)}\right)\right]^{\frac{p}{\alpha}}\right| d \nu .
\end{aligned}
$$

Since $\varphi(\lambda), \lambda \in \Pi^{n}$, is continuous at the point $\lambda^{(0)}$, then for all $\epsilon_{1}>0$ there exists $\delta>0$, such that for any $x,\|x\| \leq \delta$, it can be written

$$
\left|\varphi\left(\lambda^{(0)}+x\right)-\varphi\left(\lambda^{(0)}\right)\right| \leq \epsilon_{1}
$$

Therefore, for any $\delta_{1}, 0<\delta_{1}<\delta<\pi$,

$$
\begin{gathered}
\int_{\Pi^{n}} W_{T}(\nu)\left|\left[\psi_{T}\left(\lambda^{(0)}+\nu\right)\right]^{\frac{p}{\alpha}}-\left[\varphi\left(\lambda^{(0)}\right)\right]^{\frac{p}{\alpha}}\right| d \nu= \\
=\int_{\|\nu\| \leq \delta_{1}} W_{T}(\nu)\left|\left[\psi_{T}\left(\lambda^{(0)}+\nu\right)\right]^{\frac{p}{\alpha}}-\left[\varphi\left(\lambda^{(0)}\right)\right]^{\frac{p}{\alpha}}\right| d \nu+ \\
+\int_{\Pi^{n} \backslash\left\{\|\nu\| \leq \delta_{1}\right\}} W_{T}(\nu)\left|\left[\psi_{T}\left(\lambda^{(0)}+\nu\right)\right]^{\frac{p}{\alpha}}-\left[\varphi\left(\lambda^{(0)}\right)\right]^{\frac{p}{\alpha}}\right| d \nu=A_{1}+A_{2},
\end{gathered}
$$

where

$$
\begin{gathered}
A_{1}=\int_{\|\nu\| \leq \delta_{1}} W_{T}(\nu)\left|\left[\psi_{T}\left(\lambda^{(0)}+\nu\right)\right]^{\frac{p}{\alpha}}-\left[\varphi\left(\lambda^{(0)}\right)\right]^{\frac{p}{\alpha}}\right| d \nu \\
A_{2}=\int_{\Pi^{n} \backslash\left\{\|\nu\| \leq \delta_{1}\right\}} W_{T}(\nu)\left|\left[\psi_{T}\left(\lambda^{(0)}+\nu\right)\right]^{\frac{p}{\alpha}}-\left[\varphi\left(\lambda^{(0)}\right)\right]^{\frac{p}{\alpha}}\right| d \nu .
\end{gathered}
$$

Consider the item $A_{1}$. By the inequality

$$
\left|x^{q}-y^{q}\right| \leq \frac{q}{2}|x-y|\left(x^{q-1}+y^{q-1}\right)
$$

valid for $x, y>0, q \in(0,1], q \geq 2$, and assuming $x=\psi_{T}\left(\lambda^{(0)}+\nu\right), y=\varphi\left(\lambda^{(0)}\right), q=\frac{\alpha}{p}$, we obtain

$$
\begin{gathered}
A_{1}=\int_{\|\nu\| \leq \delta_{1}} W_{T}(\nu)\left|\left[\psi_{T}\left(\lambda^{(0)}+\nu\right)\right]^{\frac{p}{\alpha}}-\left[\varphi\left(\lambda^{(0)}\right)\right]^{\frac{p}{\alpha}}\right| d \nu \leq \\
\leq \max _{\|\nu\| \leq \delta_{1}}\left|\left[\psi_{T}\left(\lambda^{(0)}+\nu\right)\right]^{\frac{p}{\alpha}}-\left[\varphi\left(\lambda^{(0)}\right)\right]^{\frac{p}{\alpha}}\right| \int_{\|\nu\| \leq \delta_{1}} W_{T}(\nu) d \nu \leq \\
\leq \max _{\|\nu\| \leq \delta_{1}} \frac{p}{2 \alpha}\left|\left[\psi_{T}\left(\lambda^{(0)}+\nu\right)\right]^{\frac{p}{\alpha}-1}+\left[\varphi\left(\lambda^{(0)}\right)\right]^{\frac{p}{\alpha}-1}\right|\left|\psi_{T}\left(\lambda^{(0)}+\nu\right)-\varphi\left(\lambda^{(0)}\right)\right|= \\
=C_{1} \max _{\|\nu\| \leq \delta_{1}}\left|\psi_{T}\left(\lambda^{(0)}+\nu\right)-\varphi\left(\lambda^{(0)}\right)\right|,
\end{gathered}
$$

where

$$
C_{1}=\max _{\nu \in \Pi^{n}} \frac{p}{2 \alpha}\left|\left[\psi_{T}\left(\lambda^{(0)}+\nu\right)\right]^{\frac{p}{\alpha}-1}+\left[\varphi\left(\lambda^{(0)}\right)\right]^{\frac{p}{\alpha}-1}\right|<\infty .
$$

By (16), we have

$$
\max _{\|\nu\| \leq \delta_{1}}\left|\psi_{T}\left(\lambda^{(0)}+\nu\right)-\varphi\left(\lambda^{(0)}\right)\right|=
$$




$$
\begin{aligned}
& =\left.\max _{\|\nu\| \leq \delta_{1}}\left|\int_{\Pi^{n}}\right| H_{T}(\mu)\right|^{\alpha} \varphi\left(\lambda^{(0)}+\nu+\mu\right) d \mu-\varphi\left(\lambda^{(0)}\right) \mid \leq \\
& \leq \max _{\|\nu\| \leq \delta_{1}} \int_{\Pi^{n}}\left|H_{T}(\mu)\right|^{\alpha}\left|\varphi\left(\lambda^{(0)}+\nu+\mu\right)-\varphi\left(\lambda^{(0)}\right)\right| d \mu .
\end{aligned}
$$

For any fixed $\delta_{2}, 0<\delta_{2}<\delta<\pi, 0<\delta_{1}+\delta_{2}<\delta$, we obtain

$$
\begin{gathered}
\max _{\|\nu\| \leq \delta_{1}} \int_{\Pi^{n}}\left|H_{T}(\mu)\right|^{\alpha}\left|\varphi\left(\lambda^{(0)}+\nu+\mu\right)-\varphi\left(\lambda^{(0)}\right)\right| d \mu= \\
=\max _{\|\nu\| \leq \delta_{1}} \int_{\|\mu\| \leq \delta_{2}}\left|H_{T}(\mu)\right|^{\alpha}\left|\varphi\left(\lambda^{(0)}+\nu+\mu\right)-\varphi\left(\lambda^{(0)}\right)\right| d \mu+ \\
+\max _{\|\nu\| \leq \delta_{1}} \int_{\Pi^{n} \backslash\left\{\|\mu\| \leq \delta_{2}\right\}}\left|H_{T}(\mu)\right|^{\alpha}\left|\varphi\left(\lambda^{(0)}+\nu+\mu\right)-\varphi\left(\lambda^{(0)}\right)\right| d \mu \leq \\
\leq \max _{\|\nu\| \leq \delta_{1}} \max _{\|\mu\| \leq \delta_{2}}\left|\varphi\left(\lambda^{(0)}+\nu+\mu\right)-\varphi\left(\lambda^{(0)}\right)\right| \int_{\|\mu\| \leq \delta_{2}}\left|H_{T}(\mu)\right|^{\alpha} d \mu+ \\
+\max _{\|\nu\| \leq \delta_{1}} \max _{\mu \in \Pi^{n}}\left|\varphi\left(\lambda^{(0)}+\nu+\mu\right)-\varphi\left(\lambda^{(0)}\right)\right| \int_{\Pi^{n} \backslash\left\{\|\mu\| \leq \delta_{2}\right\}}\left|H_{T}(\mu)\right|^{\alpha} d \mu \leq \\
\leq \max _{\|\nu\| \leq \delta_{1}\|\mu\| \leq \delta_{2}}\left|\varphi\left(\lambda^{(0)}+\nu+\mu\right)-\varphi\left(\lambda^{(0)}\right)\right|+ \\
+\max _{\|\nu\| \leq \delta_{1}} \max _{\mu \in \Pi^{n}}\left|\varphi\left(\lambda^{(0)}+\nu+\mu\right)-\varphi\left(\lambda^{(0)}\right)\right| \int_{\Pi^{n} \backslash\left\{\|\mu\| \leq \delta_{2}\right\}}\left|H_{T}(\mu)\right|^{\alpha} d \mu .
\end{gathered}
$$

By the ineqiality

$$
\|\nu+\mu\| \leq\|\nu\|+\|\mu\| \leq \delta_{1}+\delta_{2} \leq \delta,
$$

and (17),

$$
\max _{\|\nu\| \leq \delta_{1}} \max _{\|\mu\| \leq \delta_{2}}\left|\varphi\left(\lambda^{(0)}+\nu+\mu\right)-\varphi\left(\lambda^{(0)}\right)\right| \leq \epsilon_{1} .
$$

Since the function $\varphi(\lambda), \lambda \in \Pi^{n}$ is bounded on $\Pi^{n}$, then

$$
C_{2}=\max _{\|\nu\| \leq \delta_{1}} \max _{\mu \in \Pi^{n}}\left|\varphi\left(\lambda^{(0)}+\nu+\mu\right)-\varphi\left(\lambda^{(0)}\right)\right|<\infty .
$$

As far as $\left|H_{T}(\mu)\right|^{\alpha}, \mu \in \Pi^{n}$, is a kernet on $\Pi^{n}$, then for a given $\epsilon_{1}$ there exists a vector $T^{(1)}=\left(T_{1}^{(1)}, T_{2}^{(1)}, \ldots, T_{n}^{(1)}\right)$, such that for all $T \geq T^{(1)}$ the inequality $\int_{\Pi^{n} \backslash\left\{\|\mu\| \leq \delta_{2}\right\}}\left|H_{T}(\mu)\right|^{\alpha} d \mu \leq \epsilon_{1}$ holds. Then for all $T \geq T^{(1)}$,

$$
\max _{\|\nu\| \leq \delta_{1}} \max _{\mu \in \Pi^{n}}\left|\varphi\left(\lambda^{(0)}+\nu+\mu\right)-\varphi\left(\lambda^{(0)}\right)\right| \int_{\Pi^{n} \backslash\left\{\|\mu\| \leq \delta_{2}\right\}}\left|H_{T}(\mu)\right|^{\alpha} d \mu \leq C_{2} \epsilon_{1} .
$$

Consider the item $A_{2}$.

$$
\begin{aligned}
& A_{2}=\int_{\Pi^{n} \backslash\left\{\|\nu\| \leq \delta_{1}\right\}} W_{T}(\nu)\left|\left[\psi_{T}\left(\lambda^{(0)}+\nu\right)\right]^{\frac{p}{\alpha}}-\left[\varphi\left(\lambda^{(0)}\right)\right]^{\frac{p}{\alpha}}\right| d \nu \leq \\
& \leq \max _{\nu \in \Pi^{n}}\left|\left[\psi_{T}\left(\lambda^{(0)}+\nu\right)\right]^{\frac{p}{\alpha}}-\left[\varphi\left(\lambda^{(0)}\right)\right]^{\frac{p}{\alpha}}\right| \int_{\Pi^{n} \backslash\|\nu\| \leq \delta_{1}} W_{T}(\nu) d \nu .
\end{aligned}
$$


Since the functions $\psi_{T}(\lambda), \lambda \in \Pi^{n}$, and $\varphi(\lambda), \lambda \in \Pi^{n}$, are bounded on $\Pi^{n}$, then

$$
C_{3}=\max _{\nu \in \Pi^{n}}\left|\left[\psi_{T}\left(\lambda^{(0)}+\nu\right)\right]^{\frac{p}{\alpha}}-\left[\varphi\left(\lambda^{(0)}\right)\right]^{\frac{p}{\alpha}}\right|<\infty .
$$

As far as $W_{T}(\nu), \nu \in \Pi^{n}$, is a kernel on $\Pi^{n}$, then for a given $\epsilon_{1}$ there exists a vector $T^{(2)}=\left(T_{1}^{(2)}, T_{2}^{(2)}, \ldots, T_{n}^{(2)}\right)$ such, that for all $T \geq T^{(2)}$ inequality $\int_{\Pi^{n} \backslash\left\{\|\nu\| \leq \delta_{1}\right\}} W_{T}(\nu) d \nu \leq \epsilon_{1}$ holds. Then, for all $T \geq T^{(2)}$,

$$
A_{2} \leq C_{3} \epsilon_{1}
$$

By (20), (22) and (24) for a given $\epsilon_{1}>0$ there exists the vector $T_{\max }=\max \left\{T^{(1)}, T^{(2)}\right\}$, that for all $T \geq T_{\max }$, it can be written $\left|E \widehat{f}_{T}\left(\lambda^{(0)}\right)-\left[\varphi\left(\lambda^{(0)}\right)\right]^{\frac{p}{\alpha}}\right|=A_{1}+A_{2} \leq C_{1} \epsilon_{1}+C_{1} C_{2} \epsilon_{1}+C_{3} \epsilon_{1}=\left(C_{1}+C_{1} C_{2}+C_{3}\right) \epsilon_{1}$,

where $C_{1}, C_{2}, C_{3}$ are given by (19), (21), (23), respectively. Suppose, that $\epsilon=\frac{\epsilon_{1}}{C_{1}+C_{1} C_{2}+C_{3}}$ then for any $\epsilon>0$ there exists $T(\epsilon)=T_{\max }$ such that for all $T \geq T(\epsilon)$ inequality $\left|E \widehat{f}_{T}\left(\lambda^{(0)}\right)-\left[\varphi\left(\lambda^{(0)}\right)\right]^{\frac{p}{\alpha}}\right| \leq \epsilon$ holds, i.e.

$$
E \widehat{f}_{T}\left(\lambda^{(0)}\right) \underset{T \rightarrow \infty}{\longrightarrow}\left[\varphi\left(\lambda^{(0)}\right)\right]^{\frac{p}{\alpha}}
$$

Consider the variance of the estimation (11). We divide the coordinate $j$ of the parallelepiped $\Pi^{n}$ into $L_{T_{j}}$ equal parts. These partitionings generate the partitioning of the parallelepiped $\Pi^{n}$ into $L_{T}$ parts. Let $Q_{s}$ be the parallelepiped with number $s$ and let $\nu^{(s)}$ is some point belonging to $Q_{s}, s=\overline{1, L_{T}}$.

We have

$$
\begin{gathered}
\operatorname{Var} \widehat{f}_{T}\left(\lambda^{(0)}\right)=\operatorname{Var}\left(\int_{\Pi^{n}} W_{T}(\nu) I_{T}\left(\lambda^{(0)}+\nu\right) d \nu\right) \cong \\
\cong \operatorname{Var}\left(\sum_{s=1}^{L_{T}} W_{T}\left(\nu^{(s)}\right) I_{T}\left(\lambda^{(0)}+\nu^{(s)}\right) \frac{(2 \pi)^{n}}{L_{T}}\right)= \\
=\sum_{s=1}^{L_{T}} \operatorname{Var}\left(W_{T}\left(\nu^{(s)}\right) I_{T}\left(\lambda^{(0)}+\nu^{(s)}\right) \frac{(2 \pi)^{n}}{L_{T}}\right) \text { as } T \rightarrow \infty .
\end{gathered}
$$

As it follows from Orlova (1993), we have

$$
\operatorname{Var}_{T}(\lambda)=V(p, \alpha)\left[\psi_{T}(\lambda)\right]^{\frac{2 p}{\alpha}}, \lambda \in \Pi^{n}
$$

where $V(p, \alpha)=\frac{(k(p, \alpha))^{2}}{k(2 p, \alpha)}-1$ and where $\psi_{T}(\lambda), \lambda \in \Pi^{n}$, is given by (16). By substituting (27) into (26), we can write

$$
\operatorname{Var} \widehat{f}_{T}\left(\lambda^{(0)}\right) \cong\left(\frac{(2 \pi)^{n}}{L_{T}}\right)^{2} \sum_{s=1}^{L_{T}} W_{T}^{2}\left(\nu^{(s)}\right) V(p, \alpha)\left[\psi_{T}\left(\lambda^{(0)}+\nu^{(s)}\right)\right]^{\frac{2 p}{\alpha}}+
$$




$$
\begin{gathered}
+\left(\frac{(2 \pi)^{n}}{L_{T}}\right)^{2} \sum_{s=1}^{L_{T}} \sum_{r=1, s \neq r}^{L_{T}} W_{T}\left(\nu^{(s)}\right) W_{T}\left(\nu^{(r)}\right) \operatorname{cov}\left\{I_{T}\left(\lambda^{(0)}+\nu^{(s)}\right), I_{T}\left(\lambda^{(0)}+\nu^{(r)}\right)\right\} \leq \\
\leq \frac{(2 \pi)^{n}}{L_{T}} \int_{\Pi^{n}} W_{T}^{2}(\nu) V(p, \alpha)\left[\psi_{T}\left(\lambda^{(0)}+\nu\right)\right]^{\frac{2 p}{\alpha}} d \nu+ \\
+\max _{s, r=\frac{1, L_{T}}{s} \neq r} \operatorname{cov}\left\{I_{T}\left(\lambda^{(0)}+\nu^{(s)}\right), I_{T}\left(\lambda^{(0)}+\nu^{(r)}\right)\right\} \times \\
\quad \times\left(\frac{(2 \pi)^{n}}{L_{T}}\right)^{2} \sum_{s=1}^{L_{T}} \sum_{r=1, s \neq r}^{L_{T}} W_{T}\left(\nu^{(s)}\right) W_{T}\left(\nu^{(r)}\right) .
\end{gathered}
$$

Since

$$
V(p, \alpha)\left[\psi_{T}\left(\lambda^{(0)}+\nu\right)\right]^{\frac{2 p}{\alpha}}<\infty \text { and } \frac{(2 \pi)^{n}}{L_{T}} \int_{\Pi^{n}} W_{T}^{2}(\nu) d \nu \cong \frac{M_{T}}{L_{T}} \int_{R^{n}} w_{T}^{2}(x) d x
$$

as $T \longrightarrow \infty$, we have

$$
\frac{(2 \pi)^{n}}{L_{T}} \int_{\Pi^{n}} W_{T}^{2}(\nu) V(p, \alpha)\left[\psi_{T}\left(\lambda^{(0)}+\nu\right)\right]^{\frac{2 p}{\alpha}} d \nu=O\left(\frac{M_{T}}{L_{T}}\right) \text { as } T \longrightarrow \infty .
$$

by (6) and by the properties of the sequences $L_{T_{j}}$ and $M_{T_{j}}, j=\overline{1, n}$.

Consider

$$
\begin{gathered}
\left(\frac{(2 \pi)^{n}}{L_{T}}\right)^{2} \sum_{s=1}^{L_{T}} \sum_{r=1, s \neq r}^{L_{T}} W_{T}\left(\nu^{(s)}\right) W_{T}\left(\nu^{(r)}\right) \leq\left(\frac{(2 \pi)^{n}}{L_{T}}\right)^{2} \sum_{s=1}^{L_{T}} \sum_{r=1}^{L_{T}} W_{T}\left(\nu^{(s)}\right) W_{T}\left(\nu^{(r)}\right)= \\
=\left(\frac{(2 \pi)^{n}}{L_{T}} \sum_{s=1}^{L_{T}} W_{T}\left(\nu^{(s)}\right)\right)^{2} \cong\left(\int_{\Pi^{n}} W_{T}(\nu) d \nu\right)^{2}=1 \text { as } T \longrightarrow \infty .
\end{gathered}
$$

As it follows from Demesh and Chekhmenok (2004a), we have

$\operatorname{cov}\left\{I_{T}\left(\lambda^{(0)}+\nu^{(s)}\right), I_{T}\left(\lambda^{(0)}+\nu^{(t)}\right)\right\}=O\left(\int_{\Pi^{n}}\left|H_{T}\left(\nu^{(s)}-\nu\right) H_{T}\left(\nu^{(r)}-\nu\right)\right|^{\frac{\alpha}{2}} d \nu\right)$.

By (28), we then obtain

$$
\operatorname{Var} \widehat{f}_{T}\left(\lambda^{(0)}\right) \underset{T \rightarrow \infty}{\longrightarrow} 0 .
$$

To prove (14), we show that $P\left(\left|\widehat{\varphi}_{T}\left(\lambda^{(0)}\right)-\varphi\left(\lambda^{(0)}\right)\right|>a\right) \longrightarrow 0$ as $T \longrightarrow \infty$ for any $a>0$. Let $f\left(\lambda^{(0)}\right)=\left[\varphi\left(\lambda^{(0)}\right)\right]^{\frac{p}{\alpha}}$. Assuming in the inequality (18) $x=\widehat{f}_{T}\left(\lambda^{(0)}\right)$, $y=f\left(\lambda^{(0)}\right), q=\frac{\alpha}{p}$, we obtain

$$
\begin{gathered}
\left|\widehat{\varphi}_{T}\left(\lambda^{(0)}\right)-\varphi\left(\lambda^{(0)}\right)\right|=\left|\left[\widehat{f}_{T}\left(\lambda^{(0)}\right)\right]^{\frac{\alpha}{p}}-\left[f\left(\lambda^{(0)}\right)\right]^{\frac{\alpha}{p}}\right| \leq \\
\leq \frac{\alpha}{2 p}\left|\widehat{f}_{T}\left(\lambda^{(0)}\right)-f\left(\lambda^{(0)}\right)\right|\left(\left[\widehat{f}_{T}\left(\lambda^{(0)}\right)\right]^{\frac{\alpha}{p}-1}+\left[f\left(\lambda^{(0)}\right)\right]^{\frac{\alpha}{p}-1}\right),
\end{gathered}
$$

By (25) and (29), $E\left|\widehat{f}_{T}\left(\lambda^{(0)}\right)-f\left(\lambda^{(0)}\right)\right|^{2}=D \widehat{f}_{T}\left(\lambda^{(0)}\right)+\left(E \widehat{f}_{T}\left(\lambda^{(0)}\right)-f\left(\lambda^{(0)}\right)\right)^{2} \rightarrow$ 0 as $T \longrightarrow \infty$. Therefore 
$\left[\widehat{f}_{T}\left(\lambda^{(0)}\right)\right]^{\frac{\alpha}{p}-1}+\left[f\left(\lambda^{(0)}\right)\right]^{\frac{\alpha}{p}-1} \stackrel{P}{\longrightarrow} 2\left[f\left(\lambda^{(0)}\right)\right]^{\frac{\alpha}{p}-1}$ as $T \longrightarrow \infty$. Using the Chebyshev inequality for any $a>0$ we have:

$$
P\left(\left|\widehat{\varphi}_{T}\left(\lambda^{(0)}\right)-\varphi\left(\lambda^{(0)}\right)\right|>a\right) \leq \text { Const } \frac{E\left|\widehat{f}_{T}\left(\lambda^{(0)}\right)-f\left(\lambda^{(0)}\right)\right|^{2}}{a^{2}} \longrightarrow 0 .
$$

as $T \longrightarrow \infty$. Theorem is proved.

\section{References}

D.R. Brillinger. Time Series: Data Analysis and Theory. Holt Rinehart and Winston, Inc., New York: Holt, 1975.

N.N Demesh and S.L. Chekhmenok. Asymptotic behaviour of smoothed periodogram of discrete-time symmetric stable homogeneous random field. Preceedings of National Academy of Science of Belarus, ser.phys.-math. sciences, 4:47-50, 2004a.

N.N Demesh and S.L. Chekhmenok. Using of smoothed periodogram in evaluation of spectrum of discrete-time stable random processes. Preceedings of Belarus State University, ser 1: phys.math.inf., 1:59-63, 2004b.

C.D. Hardin. On the spectral representation of symmetric stable processes. Journal of the Multivariate Analysis, (12):385-401, 1982.

N.N. Leonenko and A.V. Ivanov. Statistical Analysis of Random Fields. Kluwer Academic Publishers., Boston, 1989.

J.P. Nolan. Path properties of index- $\beta$ stable fields. The Annals of Probability, 16(4): 1569-1607, 1988.

E.N. Orlova. Investigation of periodogram statistics of spectral density of a homogeneous stable random field. Proceedings of the conference "Modern problems of computer data analysis and modeling, 1:75-79, 1993.

A.V. Skorokhod. Random processes with independent increments. Kluwer Academic Publishers., Boston, 1989.

N.N. Trush. Asymptotical properties of time series. BSU, Minsk, 1999.

N.N. Trush and E.N. Orlova. Statistical properties of the periodogram of stable random field. Preceedings of National Academy of Science of Belarus, ser.phys.-math. sciences, 2:33-41, 1994.

V.M. Zolotarev. One-dimensional Stable Distributions. American Mathematical Society: Translations of Mathematical Monographs., 1986. 
Corresponding author's address:

Dr. Nikolay Demesh

Department of Applied Mathematics

Belarusian State University

Fr. Skoriny av. 4

220050 Minsk

Belarus

Tel. +375 172095522

E-mail: Demesh@bsu.by 